\title{
Coexistence of an endocrine tumour in a serous cystadenoma (microcystic adenoma) of the pancreas, an unusual association
}

\author{
M Ö Üstün, N Tuğyan, M Tunakan
}

\begin{abstract}
A pancreatic endocrine tumour arising within a serous cystadenoma is reported. A 49 year old woman was admitted with a history of epigastric pain, nausea, vomiting, and weight loss of two months duration. She had been diabetic for 12 years. An epigastric mass was palpated in the physical examination, and computed tomography revealed a multiloculated cystic lesion in the pancreas. Pathological examination of the pancreatic tumour revealed the coexistence of a serous cystadenoma and an endocrine tumour. The endocrine tumour, which was located inside the serous cystadenoma, was $1 \mathrm{~cm}$ in diameter. The first case of a serous cystadenoma of the pancreas containing a pancreatic endocrine tumour was reported in the literature recently. This paper reports another incidentally found pancreatic endocrine tumour arising within a serous cystadenoma. (F Clin Pathol 2000;53:800-802)
\end{abstract}

Keywords: pancreatic endocrine tumour; serous cystadenoma; cystic neoplasms of the pancreas

Serous cystadenomas, also known as microcystic adenomas or glycogen rich adenomas, are benign tumours of the pancreas, which occur in elderly patients and have a slight female preponderance. ${ }^{12}$ These tumours are either discovered incidentally or present as an abdominal mass with or without local pain. If the mass occurs in the pancreatic head, it can result in gastrointestinal or biliary tract obstruction, and when sufficient islet cell tissue is destroyed by the tumour, diabetes mellitus may also occur. ${ }^{1}$ Although these tumours usually arise in normal pancreas, rarely they are associated with other pancreatic neoplasms, such as pancreatic ductal adenocarcinoma. ${ }^{3}$ There is only one report in the literature on the coexistence of a pancreatic endocrine tumour (PET) and a serous cystadenoma. ${ }^{4}$ In this article, we report another patient presenting with a PET and a serous cystadenoma.

\section{Case report}

A 49 year old woman was admitted to hospital because of epigastric pain, nausea, vomiting, and weight loss. She had a history of diabetes mellitus for 12 years. Physical examination revealed a palpable epigastric mass. Computed tomography scan showed a multiloculated cystic mass measuring $12 \mathrm{~cm}$ in diameter within the head and the body of the pancreas. Invasion to adjacent structures was not seen. There was central scarring with calcifications in the central region of the mass. Biochemical analyses including amylase and bilirubin were within the normal ranges except for raised alkaline phosphatase (1786 U/litre; normal range, 64-306) and alanine aminotransferase (190 U/litre; normal range, 4-37). In addition, the patient was hyperglycaemic (fasting plasma glucose concentration, $2770 \mathrm{mg} /$ litre; normal range, 75-115). The clinical diagnosis was a cystic pancreatic neoplasm. A modified Whipple resection was performed and portions of the small bowel $(20 \mathrm{~cm})$ and the proximal pancreas $(13 \times 10 \times 7 \mathrm{~cm})$ were resected. Nearly all of the resected pancreatic tissue was composed of a tan pink, multilocular cystic neoplasm. A central stellate scar was present. The cystic spaces were filled with a clear fluid and ranged from 0.2 to $2.5 \mathrm{~cm}$ in diameter.

The surgical specimen was fixed in $10 \%$ formalin and sections were stained with haematoxylin and eosin, periodic acid Schiff (PAS), PAS with diastase, Alcian blue $(\mathrm{pH} 2.5)$, and mucicarmine. Immunohistochemical studies were performed on paraffin wax embedded sections using a standard avidin-biotincomplex method with diaminobenzidine as the chromogen. Commercially available monoclonal antibodies against neuron specific enolase (NSE; 1/50), chromogranin (1/100), synaptophysin (1/10), epithelial membrane antigen (EMA; prediluted; Dako A/S, Denmark), LEU 7 (1/25), gastrin (1/50), insulin $(1 / 500)$, glucagon (1/100), and somatostatin $(1 / 50)$ were used.

Microscopic examination revealed multiple cysts separated by fibrous connective septa. Single layer cuboidal epithelial cells with bland nucleus and clear or eosinophilic cytoplasm lined the cysts. PAS positive intracytoplasmic material digested with diastase was consistent with glycogen (fig 1). Stains for mucin were negative in tumour cells. Although there were micropapillary structures in some areas, necrosis, nuclear atypia, and mitoses were absent. Enlarged islets were seen under the cyst epithelium. The non-neoplastic pancreas was normal in appearance; because serous cystadenomas usually grow slowly, the presence of pancreatic islets within the tumours was an expected finding. ${ }^{2}$ In addition, enlargement of islets may accompany pancreatic endocrine neoplasms. ${ }^{5}$ Inside the central satellite scar, a microscopic focus of uniform cells in solid and gyriform pattern was seen. At the periphery of
Accepted for publication 17 March 2000 


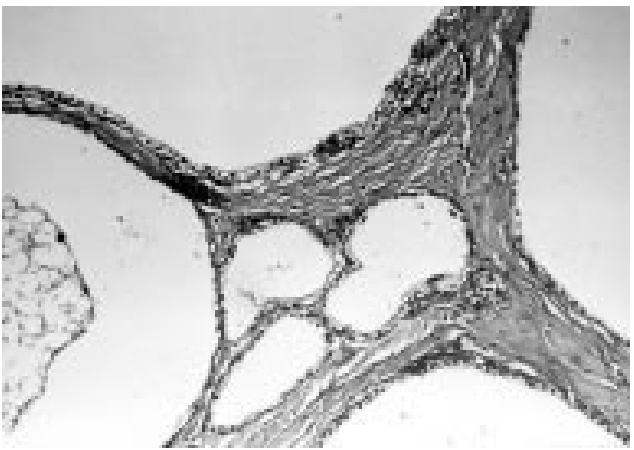

Figure 1 A single layer of cuboidal epithelium lines the cysts. The periodic acid Schiff (PAS) reaction has disappeared after diastase in the cyst epithelium (PAS with diastase).

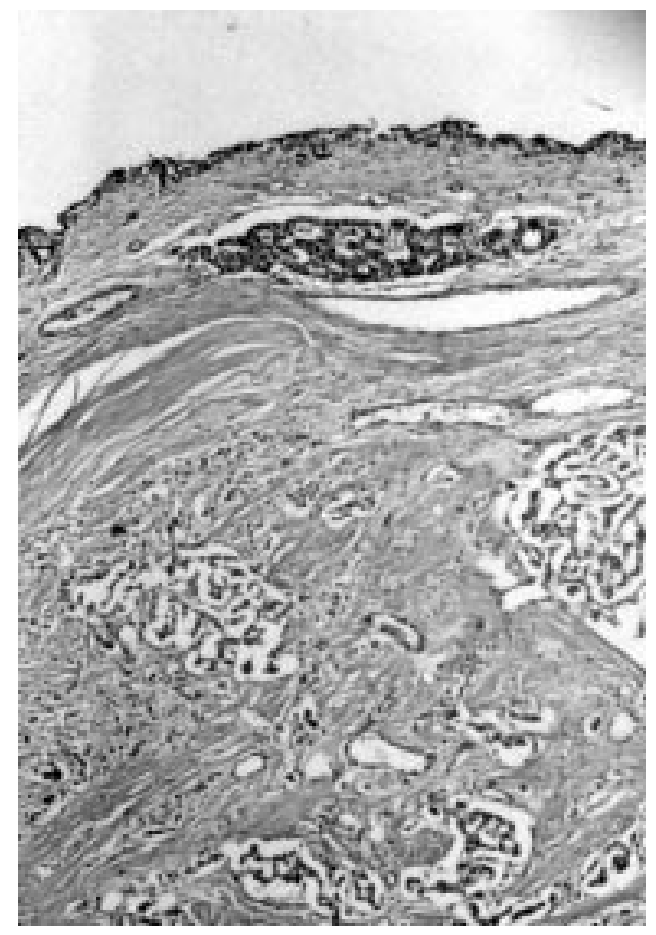

Figure 2 The periphery of the pancreatic endocrine tumour is surrounded by cyst epithelium (haematoxylin and eosin stained section).

the tumour, these uniform cells were embedded in stroma as single cell lines. Cells were small with scant eosinophilic cytoplasm and stippled nuclei, consistent with endocrine cells. The solid endocrine tumour focus was surrounded completely by the serous cystadenoma (fig 2). Immunohistochemical examination revealed strong positive staining for EMA within the serous cystadenoma component, although no such staining was seen in the endocrine tumour component. In contrast, immunohistochemical staining for NSE, chromogranin, synaptophysin, LEU 7, insulin, and glucagon was positive in the endocrine tumour, whereas all were negative in the cyst epithelium. Focal and weak staining was seen for glucagon, whereas insulin staining was focal but strong in intensity. Gastrin and somatostatin staining was not detected.

Although the diabetic status of the patient did not change after the removal of the tumour, she was well one year after surgery.

\section{Discussion}

To our knowledge, a PET arising in association with a serous cystadenoma was described first by Keel et al in 1996. The patient was a 47 year old woman with a history of systemic lupus erythematosus who had been using steroids chronically for seven years. ${ }^{4}$ Our case presented here is a 49 year old woman with a history of diabetes mellitus for 12 years.

From the embryological and histogenetic point of view, the potential of pancreatic ductal stem cells for biphasic differentiation along either glandular epithelial or neuroendocrine pathways would not be unexpected. The presence of endocrine cells within exocrine carcinomas has been reported previously in pancreatic tumours. ${ }^{6}$ Pour et al evaluated the pancreatic carcinomas of Syrian golden hamsters and reported a close relation between endocrine and ductal cells, pointing towards a common origin from a totipotent cell. According to this study, pancreatic carcinogenesis was accompanied by the proliferation of ductular formations between islets (intrainsular ductules). First an adenomatous and cystadenomatous pattern and then adenocarcinoma developed within pre-existing ductules, and within the newly formed intrainsular ductules. Simultaneously, during the process islets had gradually atrophied, but their remnants could be detected within the thin connective tissue septa of the cystadenomatous pattern. These findings suggest that both exocrine and endocrine components of the pancreas might have the same origin - the multipotential stem cell of the ductal epithelium, which originates from the endodermal epithelium.

Biphasic differentiation is seen in some pancreatic disorders, either benign or malignant. ${ }^{8-10}$ Nesidioblastosis is another example of the close histogenetic relation between the exocrine and endocrine pancreas. An unusual case of pancreatic carcinoma with both adenocarcinomatous and islet cell differentiation, along with ultrastructural evidence of focal acinar differentiation, has also been described. ${ }^{9}$

Pancreatoblastoma appears to be the counterpart of childhood tumours that arise from stem cells in other organs. Both endocrine and exocrine differentiation have been identified in component cells such that ductal, acinar, and islet cells may be seen at the histological, immunohistochemical, and ultrastructural level. ${ }^{11}$ The acinar-endocrine cell tumour of the pancreas is a rare mixed tumour reported only in one paediatric and one adult patient. It is sometimes impossible to differentiate acinar cell carcinomas from endocrine tumours by light microscopy because of the histological similarities. The detection of both zymogen and neuroendocrine granules within the same cell by ultrastructural and enzymatic analyses in acinar-endocrine cell tumours suggests a neoplastic proliferation of intermediate cells. ${ }^{8} 10$ Similarly, simultaneous neuroendocrine granule formation concentrated in the basal pole or in the cytoplasmic processes with mucin production have been described in amphicrine carcinomas (mucinous islet cell carcinomas). 
These are rare neoplasms composed of both exocrine and endocrine differentiated cells. ${ }^{12}$

In the French literature, a patient with an endocrine tumour associated with a serous cystadenoma and cystadenocarcinoma was reported, but all three tumours were located separately in the pancreas. ${ }^{3}$ Our case is composed of benign serous epithelial and neuroendocrine components. Immunohistochemical studies in our case show the evidence of divergent differentiation. The serous cystadenoma component of the tumour was strongly immunoreactive for EMA only, the endocrine tumour component was immunoreactive for NSE, chromogranin, synaptophysin, LEU 7, insulin, and glucagon. In functional endocrine neoplasms, a minor population of hormone producing cells is often present in addition to the principal cellular type, as in our case. In the current case, weak glucagon staining was seen in addition to strong insulin staining. The diabetic status of the patient despite insulin secretion by the endocrine neoplasm might be the result of excessive glucagon secretion and islet cell tissue destruction by serous cystadenoma.

In summary, this is the second case of PET arising within a serous cystadenoma in the literature. Incidentally found PETs were 1 and $1.5 \mathrm{~cm}$ in diameter in our case and in Keel and co-workers' case, respectively. Serous neoplasms are almost always benign tumours, although rare malignant examples have been reported. ${ }^{13}$ The correlation between the morphological features and the clinical behaviour of PET is poor. Only the existence of metastatic disease indicates malignancy in PET. There are no reports of patients with resected cystadenomas developing metastases from unsuspected endocrine tumours that were missed. Although both of the reported cases are well on short term follow up, the clinical relevance of this rare association is still unclear.

The authors thank Dr Paul van Diest for providing and evaluating the insulin, glucagon, gastrin, somatostatin, and LEU 7 immunohistochemical stains.

1 Alpert LC, Truong LD, Bossart MI, et al. Microcystic adenoma (serous cystadenoma) of the pancreas. A study of 14 cases with immunohistochemical and electron microscopic correlation. Am f Surg Pathol 1988;12:251-63.

2 Compagno J, Oertel JE. Microcystic adenomas of the pancreas (glycogen-rich cystadenomas). Am 7 Clin Pathol 1978;69:289-98.

3 Heresbach D, Robert 1, Le Berre N, et al. Tumeurs kystiques et tumeur endocrine du pancreas [abstract]. Gastroenterol Clin Biol 1993;17:968-71.

4 Keel SB, Zukerberg L, Graeme-Cook F, et al. A pancreatic endocrine tumor arising within a serous cystadenoma of the pancreas. Am f Surg Pathol 1996;20:471-5.

5 Klöppel G, Willemer S, Stamm B, et al. Pancreatic lesions and hormonal profile of pancreatic tumors in multiple endocrine neoplasia type 1 . An immunohistochemical study of nine patients. Cancer 1986;57:1824-32.

6 Pour P. Islet cells as a component of pancreatic ductal neoplasms. Experimental study: ductular cells, including islet cell precursors, as primary progenitor cells of tumors. $A m \mathcal{F}$ Pathol 1978;90:295-316.

7 Pour P, Althoff J, Takahashi M. Early lesions of pancreatic ductal carcinoma in the hamster model. Am f Pathol 1977; 88:291-308.

8 Ichijima K, Akaishi K, Toyoda N, et al. Carcinoma of the pancreas with endocrine component in childhood. A case report. Am f Clin Pathol 1985;83:95-100.

9 Schron DS, Mendelsohn G. Pancreatic carcinoma with duct, endocrine, and acinar differentiation. A histologic, immunocytochemical and ultrastructural study. Cancer 1984;54:1766-70.

10 Ulich T, Cheng L, Lewin K. Acinar-endocrine cell tumor of the pancreas. Cancer 1982;50:2099-105.

11 Morohoshi T, Kanda M, Horie A, et al. Immunocytochemical markers of uncommon pancreatic tumors. Acinar cell carcinoma, pancreatoblastoma, and solid cystic (papillarycystic) tumor. Cancer 1987;59:739-47.

2 Ordonez NG, Balsaver AM, Mackay B. Mucinous islet cell (amphicrine) carcinoma of the pancreas associated with watery diarrhea and hypokalemia syndrome. Hum Pathol 1988;12:1458-61.

13 Yoshimi N, Sugie S, Tanaka T, et al. A rare case of serous cystadenoma of the pancreas. Cancer 1992;69:2449-53. 